\title{
Simulation of Spraying Process in Lateral VRI Sprinkler System based on PWM
}

\author{
ZHOU Zhiyu, TAO Shuai and MO Jinqiu ${ }^{\mathrm{a}}$ \\ School of Mechanical Engineering, Shanghai Jiao Tong University, Shanghai, China
}

\begin{abstract}
Lateral VRI sprinkler system is widely used to improve water productivity nowadays. And Pulse width modulation (PWM) technique is applied to control flow rate of each sprinkler. Uniformity and accuracy of application are main indexes to evaluate performance of lateral VRI sprinkler system. Traditional approach of measuring uniformity and accuracy is catch can trials. However, the data obtained is sparse and data collection takes time and labour. In this article, a simulation algorithm is designed to find the effects of PWM parameters and speed of VRI sprinkler system on spraying results of Nelson R3000 sprinkler efficiently. Experiments indicate that PWM duty cycle affects water depth proportionally while speed has an inverse proportional effect. And indistinctive reduction of water application is found as PWM period increases. A compensation method is also put forward to minish application error caused by PWM duty cycle, period and speed. It is also found that lower speed or shorter period leads to larger water depth error but higher application uniformity. Duty cycle has insignificant positive influence on application uniformity but barely on water depth error. Besides linear transition of water depth is observed when a sprinkler passes through two different management zones in the moving direction.
\end{abstract}

\section{Introduction}

Variable rate irrigation (VRI) sprinkler system becomes more popular worldwide due to water shortage and increasing demand for food and other agricultural resources. A farmland will be divided into management zones and water need of each zone will be determined according to soil moisture, biotic stresses and so on. By using VRI sprinkler system, water need of each zone can be satisfied precisely so that water productivity is improved ${ }^{[1]}$.

VRI technology has a broader application in largesized sprinkler irrigation system. A lateral VRI sprinkler system is usually composed of several spans on which sprinklers are installed. Each span is supported by walking towers so that it can move forward. And the lateral direction is perpendicular to the moving direction.

A commonly used method to regulate sprinkler flow is controlling the "on/off" cycle by hydraulic valve, which is also called pulse width modulation (PWM) ${ }^{[2]}$. Combined with technology of constant pressure water supply, the working pressure of sprinkler will not be changed but its flow can be regulated by adjusting the duty cycle of PWM signal. That means only if the size of nozzle and installation height are settled, the instantaneous spatial water distribution of single sprinkler is sustained.
Actually, PWM method has advantages over other irrigation methods, such as uniform application and bimodel sequencing, in terms of mean water application and uniformity ${ }^{[3]}$. However, the duty cycle, period of PWM and speed of VRI sprinkler system will affect application accuracy and uniformity ${ }^{[4]}$. The water distribution pattern also varies a lot under different factor setups ${ }^{[5]}$. To find out effects of the above factors, catch can trials are performed in these researches. Nonetheless because of the relative large size of catch can and discrete spacing between cans, there will not be too many measuring points especially when large-scale experiments are carried out. That may lead to data loss of water depth. Performing such experiments in farmland also consumes large amounts of time and labour.

In this article, feature of water depth accumulation is analysed and based on this a simulation program is developed to find out effects of duty cycle, period of PWM and speed of VRI sprinkler system on spraying results with much denser measuring points and higher efficiency.

The work described in this paper was fully supported by a grant from National Key R\&D Program of China (No. 2017YFD0700504-2).

\section{Calculation of water depth}

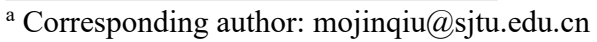


As lateral VRI sprinkler system moves forward, the round wet area of a sprinkler also sweeps forward and the water depth of a point in the moving direction is the accumulation of water volume in different position of sprinkler wet area in the time dimension. In order to control water depth accurately, it is necessary to determine the relationship between water depth and PWM parameters, speed of VRI sprinkler system, spraying feature of single sprinkler and so forth.

\subsection{Analysis of water accumulation}

Divide farmland and the wet area of single sprinkler into small blocks, which have the size of $\Delta L \cdot \Delta H$. Suppose there is a block $A$ of farmland in the moving direction, after the whole wet area passes block $A$, the water in the corresponding blocks $B_{1} 、 B_{2}, \cdots \cdots, B_{1} n(x)$ will fall into block $A$, as Figure 1 shows, where xoy is the local coordinate system of single sprinkler, $X O Y$ is the farmland coordinate system. $n(x)$ is the number of block $B$ and it depends on the positon of block $A$ and $B$ in sprinkler local system, $x$.

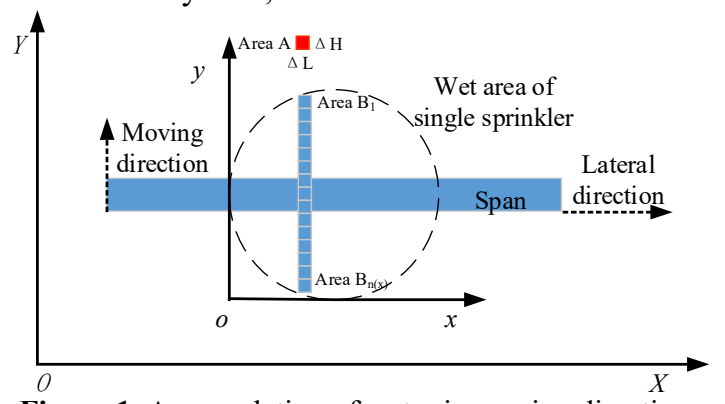

Figure 1. Accumulation of water in moving direction.

Different sprinklers will have different water distribution under different conditions. Flow density $\rho$ can be an index to describe this water distribution sufficiently and it can be defined as the flow per unit area. When the sprinkler is an ideal sprinkler, which means it will spray uniformly within its wet area, $\rho$ can be calculated as:

$$
\rho=q / 1000 \pi R^{2}
$$

Where $q$ is the sprinkler flow, $\mathrm{m}^{3} / \mathrm{h}, R$ is the radius of wet area, $\mathrm{m}$ and $\rho$ is the flow density, $\mathrm{mm} / \mathrm{h}$.

However actual sprinkler can not have a uniform distribution then the flow density $\rho$ can be regarded as a function of $x$ and $y$, denoted as $\rho(x, y)$.

$$
\rho(x, y) \begin{cases}\neq 0 & (x, y) \text { within wet area } \\ =0 & (x, y) \text { out of wet area }\end{cases}
$$

As a result, the accumulated water volume in block $A$ after the whole wet area sweeps over it can be calculated as:

$$
Q_{A}=\sum_{i=1}^{n(x)} \rho_{i} \cdot \Delta H \cdot \Delta L \cdot \Delta t
$$

Where $\rho_{i}$ is the local flow density of block $B_{i}(i=1,2, \cdots \cdots, n(x)), \Delta t$ is the time the VRI sprinkler system takes to pass through block $A$.

Then the water depth in block $A$ is:

$$
h_{A}=\frac{Q_{A}}{\Delta H \cdot \Delta L}=\sum_{i=1}^{n(x)} \rho_{i} \cdot \Delta t
$$

If PWM is introduced to control water application, the water volume of block $B_{i}$ should be judged whether it contributes to the water volume in block $A$. Thus when block $B_{i}$ passes through block $A$, its spraying status can be defined as:

$$
\text { state }_{i}= \begin{cases}0 & \text { PWM in state of "off" } \\ 1 & \text { PWM in state of "on" }\end{cases}
$$

Then the water depth can be modified:

$$
h_{A}=\frac{Q_{A}}{\Delta H \cdot \Delta L}=\sum_{i=1}^{n(x)} \rho_{i} \cdot \text { state }_{i} \cdot \Delta t
$$

When $\Delta L$ and $\Delta H$ are small enough, the VRI sprinkler system will move continuously. Then $h_{A}$ can be rewritten as:

$$
h_{A}=\int_{0}^{t_{\text {total }}} \rho(x, y) \operatorname{state}(x, y) d t
$$

Where $t_{\text {total }}$ is the time the whole wet area takes to pass through block $A$. Also apparently, $y$ is a function of time $t$.

When the VRI sprinkler system moves at an even speed $v$,

$$
\begin{gathered}
t_{\text {total }}=2 R / v \\
y=v t
\end{gathered}
$$

Specially, many sequential block $A$ will compose a management zone, as the solid area shows in Figure 2(a). The water depth in management zone can be calculated by applying Equation (7). However, for the convenience of changing sprinkler flow rate to meet different water needs of different management zones along the moving direction, the center of wet area is usually considered as a benchmark of start and end of a management. Thus an actual management area will be the dotted-line area in Figure 2(a). Since the solid area is swept by the whole wet area, it can be called completely-swept area; the dotted-line area is only swept by part of wet area so it will be called incompletely-swept area.

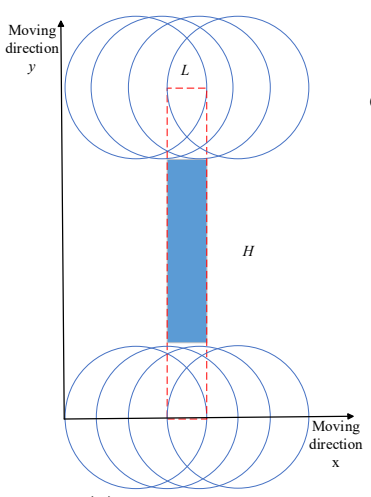

(a) Management zone

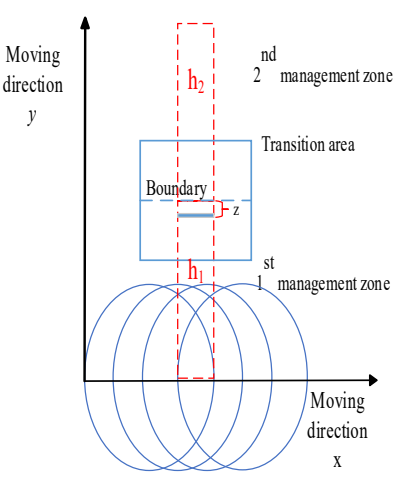

(b) Transition area
Figure 2. Management zone and transition area.

When the center of wet area arrives at the end of a management zone, which is also the start of the next management zone, the flow of sprinkler should be adjusted to meet different water needs. As a result, the water volume in range of $2 R$ around boundary (dotted line in Figure 2(b)) of management zones consists of 
water volume from two wet areas with different flow rates. Because this region results from the transition of management zones, it is called transition area (framework area in Figure 2(b)).

\subsection{Simulation algorithm flow}

To perform the water depth calculation method mentioned in section 2.1, the flow density of sprinkler should be measured first. Usually a set of rain gauges are placed along the radius of wet area to measure the radial water distribution. Then by interpolation, much more continuous radial water distribution can be obtained. Divide the wet area in Figure 1 into grid with the size of $\Delta L \cdot \Delta H$ and create a corresponding flow density matrix whose members represent for the water depth of grid. Rotate the radial water distribution around the grid center and the water depth of a block which has a certain distance from the grid center can be determined by the radial water distribution. Finally, the entire flow density matrix can be determined.

To give an expression to the spraying result after sprinklers sweep over farmland, the farmland should also be scattered into blocks with the size of $\Delta L \cdot \Delta H$. A global water distribution matrix will also be created to keep the data of water depth everywhere.

Placing the wet area grid at the appropriate location of the farmland grid is the main method to simulate spraying process. In the lateral direction, place wet area grids at a spacing which is the same with that of actual sprinklers. In the moving direction, place wet area grids at a small enough step if they are in interval of PWM "on" state; otherwise skip the interval of PWM "off" state which means placing wet area grids at a spacing the VRI sprinkler system travels during the time of PWM "off". To perform the above operation with matrix, the value of each member of flow density matrix should be passed to the right member of global water distribution matrix. To sum up, the total simulation algorithm flow is as shown in Figure 3.

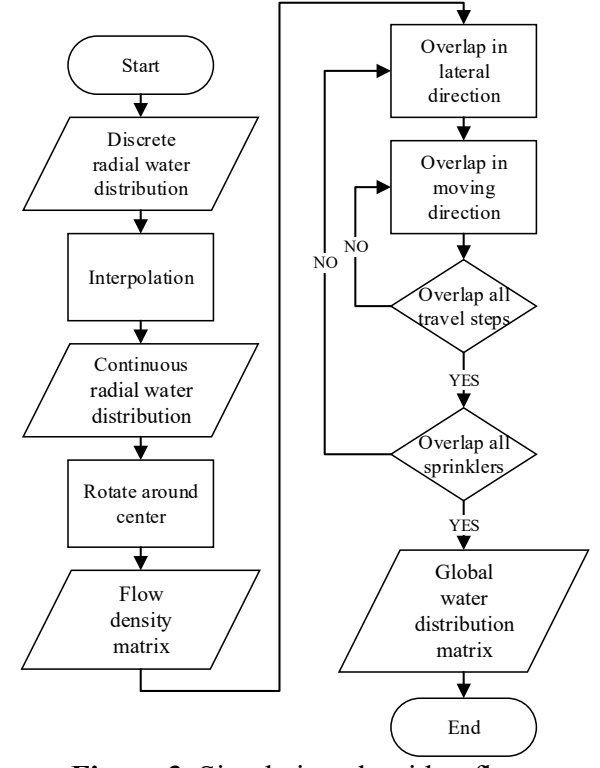

Figure 3. Simulation algorithm flow.

\section{Simulation of PWM variable rate irrigation}

The simulation algorithm designed in chapter 2 is realized in Matlab and the data adopted is discrete radial water distribution of Nelson R3000 sprinkler measured by Chen et al. under pressure of $150 \mathrm{kPa}$ and windless condition ${ }^{[6]}$. Plot the flow density matrix (Figure 4), which shows the spraying feature of sprinkler all over the wet area.

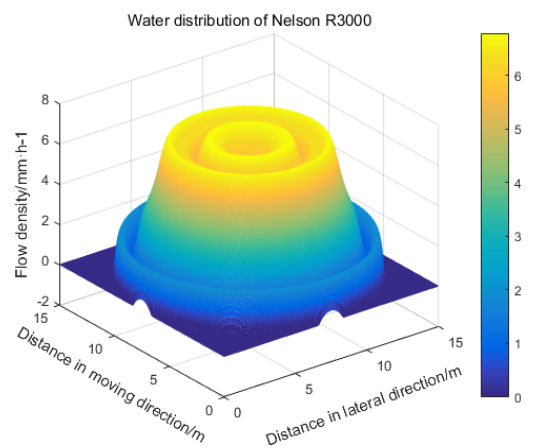

Figure 4. Water distribution of single Nelson R3000 sprinkler.

\subsection{Experiment factors setting and evaluation indexes}

The diameter of wet area of Nelson R3000 sprinkler is $15 \mathrm{~m}$. As mentioned in section 2.1, factors that will influence spraying results are duty cycle of PWM, $D$, period of PWM, $T$ and speed of VRI sprinkler system, $v$. Some indexes are proposed to evaluate spraying results:

(1) Average water depth of single sprinkler, $h$ : Average water depth of all points along lateral direction in completely-swept area;

(2) Error of water depth, $e$ : Suppose the average water depth is $h_{D}$ when the duty cycle of sprinkler is set as $D$, meanwhile the average water depth is $h_{100}$ when the duty cycle of sprinkler is set as $100 \%$. Actual duty cycle of spraying can be defined as

$$
D_{s p}=\frac{h_{D}}{h_{100}} \times 100 \%
$$

Thus

$$
e=D_{s p}-D
$$

(3) Application uniformity of multiple sprinklers: Usually the application uniformity will be better if the distance between sprinklers is between $20 \%$ and $25 \%$ of the diameter of wet area [7]. So set the distance as $d=2 R \cdot 25 \%=3.75 \mathrm{~m}$, there will be a management zone at the center of overlapped wet area and the water depth in this zone will be controlled by four sprinklers (Figure 5). 


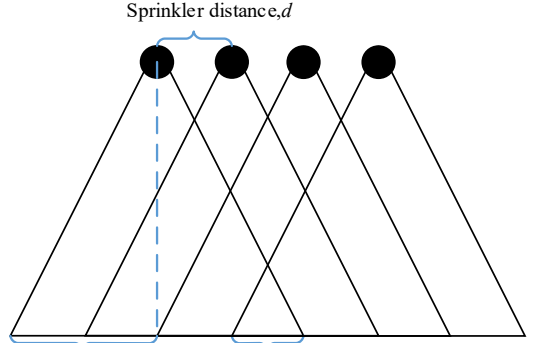

Radius of wet area, $R$ Management zone

Figure 5. Overlapped area of four sprinklers.

Application uniformity within management zone of lateral VRI sprinkler system is commonly assessed by Christiansen uniformity coefficient, $C U^{[8]}$ and lower quarter distribution uniformity coefficient, $D U{ }^{[9]}$ :

$$
\begin{gathered}
C U=\left\{1-\frac{\sum_{i=1}^{n}\left|h_{i}-\bar{h}\right|}{\sum_{i=1}^{n} h_{i}}\right\} \times 100 \% \\
D U=\frac{\overline{h^{\prime}}}{\bar{h}} \times 100 \%
\end{gathered}
$$

Where $h_{i}$ is the water depth of $i^{\text {th }}$ measuring point, $\bar{h}$ is the average water depth of points all over the measured region. $\overline{h^{\prime}}$ is the average water depth of the lowest one-quarter of the measured water depth. Compared with $C U, D U$ can prevent water depth of individual measuring points from deviating from average water depth too much, which may be caused by abnormal spraying even missed spraying.

In this article, both uniformity coefficients in the lateral direction and in the moving direction are studied. They are denoted as $C U_{l}, D U_{l}$ and $C U_{m}, D U_{m}$, respectively.

(4) Water distribution curve within transition area: The transition form of water depth within transition area will be observed when the VRI sprinkler system passes through two management zones in the moving direction.

\subsection{Results and discussion}

\subsubsection{Average water depth and its error}

To find out the effect of speed of VRI sprinkler system and PWM duty cycle, the PWM period is fixed as 60s. Speed of VRI sprinkler system varies among $0.5,1$, $2,3 \mathrm{~m} / \mathrm{min}$ and PWM duty cycle changes from $10 \%$ to $100 \%$ with a step of $10 \%$. The average water depth,

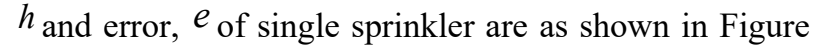
$6(\mathrm{a}),(\mathrm{b})$, respectively.
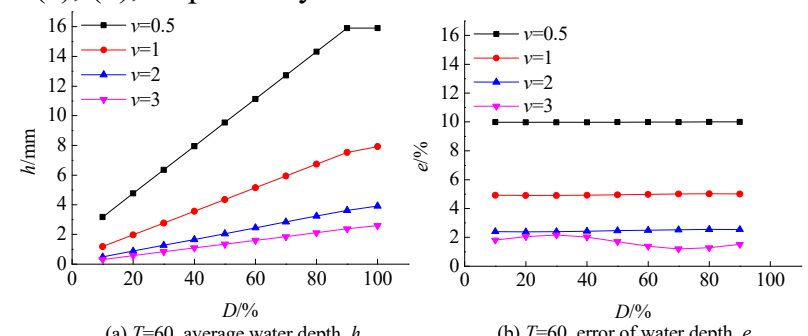

Figure 6. $T=60 \mathrm{~s}$, average water depth and error.
Fit curves in Figure 6(a) linearly in the form of $h=a v D+b($ Table 1$)$ :

Table 1. Fitting results of average water depth curves when

\begin{tabular}{|c|c|c|c|c|}
\hline \multicolumn{5}{|c}{$T=60 \mathrm{~s}}$. \\
\hline$v(\mathrm{~m} / \mathrm{min})$ & 0.5 & 1 & 2 & 3 \\
\hline$a$ & 0.0795 & 0.0790 & 0.0780 & 0.0780 \\
\hline$b$ & 1.586 & 0.387 & 0.092 & 0.057 \\
\hline
\end{tabular}

Then keep $v$ constant and $v=3 \mathrm{~m} / \mathrm{min}, T$ changes among 10,20,30,60s, The average water depth, $h$ and error, $e$ of single sprinkler are as shown in Figure 7(a), (b), respectively.
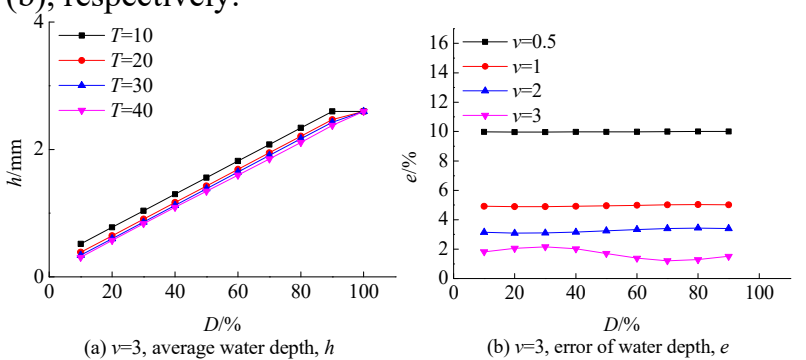

$\begin{array}{lr}\text { (a) } v=3 \text {, average water depth, } h & \text { (b) } v=3 \text {, error of water depth, } e \\ \text { Figure } 7 . v=3 \mathrm{~m} / \mathrm{min} \text {, average water depth and error }\end{array}$

Since the effect of PWM on spraying results can not show up when $D=100 \%$, only points in Figure 7(a) except for $D=100 \%$ are fitted linearly in the form of $h=a D+b$ (Table 2):

Table 2. Fitting results of average water depth curves when

\begin{tabular}{|c|c|c|c|c|}
\hline$T(\mathrm{~s})$ & 10 & 20 & 30 & 60 \\
\hline$a$ & 0.026 & 0.026 & 0.026 & 0.026 \\
\hline$b$ & 0.259 & 0.126 & 0.078 & 0.057 \\
\hline
\end{tabular}

Generally, average water depth has good linearity to the duty cycle no matter the speed changes or the PWM period changes. And it is beneficial to control water application with PWM. Table 1 shows that the slope of curve in Figure 6(a) is inversely proportional to the speed of VRI sprinkler system, which means the faster the VRI sprinkler system travels, the lower the water application is. Moreover PWM period only has a little influence on water depth since the slope of curve in Figure 7(a) does not change with it. PWM period only effects intercept of curve and water application decreases as PWM period gets longer.

Smaller speed or PWM period will attribute to the greater error of water depth, as shown in Figure 6(b) and Figure 7(b). Whereas the error varies only a little with PWM duty cycle. This indicates that a compensation method can be introduced to minish the error of water application. For example, when $v=0.5 \mathrm{~m} / \mathrm{min}, T=60 \mathrm{~s}$ or, $v=3 \mathrm{~m} / \mathrm{min}, T=10 \mathrm{~s}$, the PWM duty cycle of solenoid valve controller should be set as $70 \%$ if the water application is designed to be $80 \%$ of the maximum water supply.

\subsubsection{Uniformity of application within irrigation management zone}

Set all factors the same as section 3.2.1. Firstly, $T=60 \mathrm{~s}$ and $v=0.5,1,2,3 \mathrm{~m} / \mathrm{min}$, respectively. $D$ ranges from $10 \%$ to $100 \%$ and the step is $10 \%$. Uniformity coefficients, $C U_{l}, D U_{l}$ and $C U_{m}, D U_{m}$ are as shown in Figure 8(a), (b), (c), (d). 

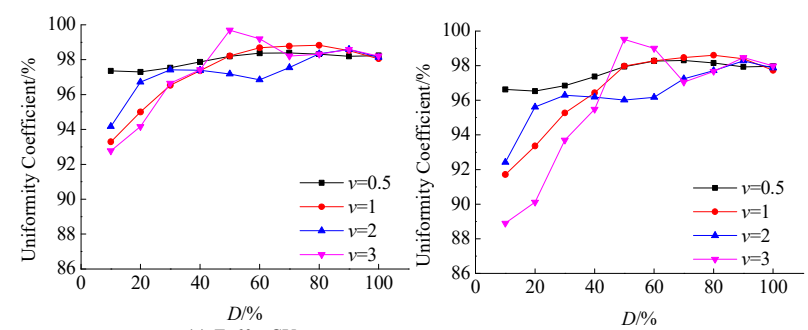
(a) $T=60, C U$
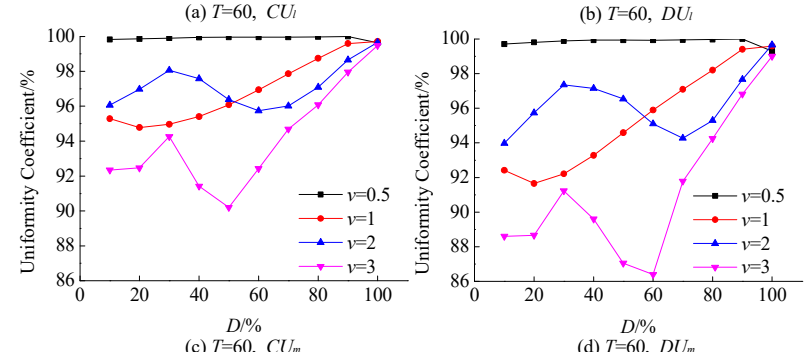

Figure 8. $T=60 \mathrm{~s}$, uniformity coefficients.

After that, keep $v$ as $3 \mathrm{~m} / \mathrm{min}$ and $T=10,20,30,60 \mathrm{~s}$, respectively. Recalculate uniformity coefficients (Figure 9).
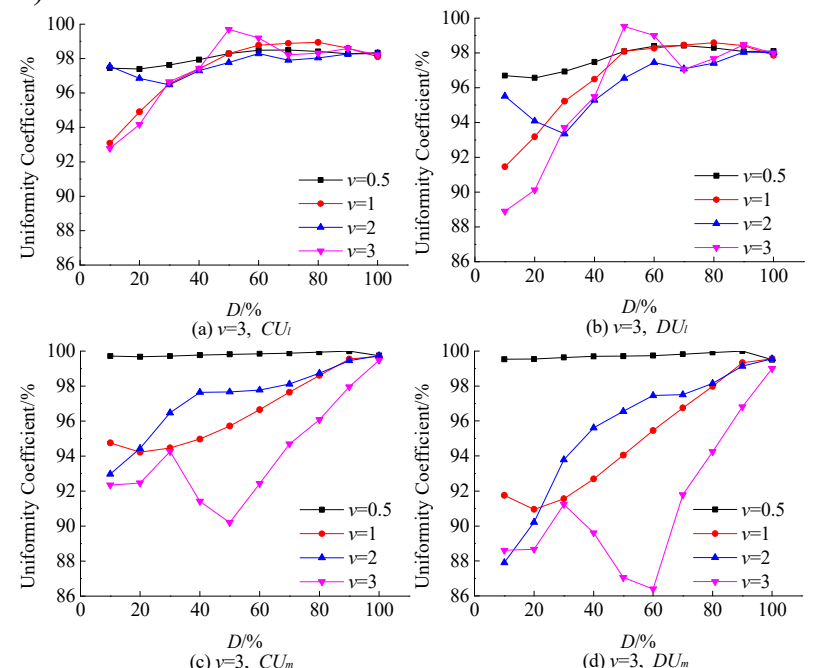

Figure 9. $v=3 \mathrm{~m} / \mathrm{min}$, uniformity coefficients

Uniformity coefficients increase with PWM duty cycle generally. That means using sprinkler under pretty low duty cycle should be avoided. Besides, as speed or PWM period increases, sharp reduction of uniformity coefficients can be observed. And uniformity coefficients will also fluctuate much more widely. Comparing Figure 8 with Figure 9, they tell that the variation of speed or PWM period has greater influence on $D U$ than on $C U$ and greater influence on uniformity coefficients in the moving direction than in the lateral direction. To obtain high enough uniformity, it is better to perform such a simulation to determine an appropriate combination of moving speed, PWM period and operating range of PWM duty cycle.

\subsubsection{Water depth distribution in transition area}

To form a transition area, make sprinkler move forward for $40 \mathrm{~m}$ and change above factors when it arrives at $20 \mathrm{~m}$. The first $20 \mathrm{~m}$ section is defined as 1 st management zone and the latter is 2 nd management zone. As a result, the entire wet area is $0 \sim 55 \mathrm{~m}, 0 \sim 15 \mathrm{~m}$ and $40 \sim 55 \mathrm{~m}$ of it are incompletely-swept area, $15 \sim 40 \mathrm{~m}$ is completely-swept area. In the completely-swept area, $20 \sim 35 \mathrm{~m}$ is transition area and the boundary is at $27.5 \mathrm{~m}$.

Factors of $1^{\text {st }}$ management zone are set as $D=90 \%$, $v=3 \mathrm{~m} / \mathrm{min}, T=20 \mathrm{~s}$ and this zone will act as a comparison. Factors of $2^{\text {nd }}$ management zone are set as four different combinations,

(1) Trial 1: $D=90 \%, v=3 \mathrm{~m} / \mathrm{min}, T=20 \mathrm{~s}$,

(2) Trial 2: $D=10 \%, v=3 \mathrm{~m} / \mathrm{min}, T=20 \mathrm{~s}$,

(3) Trial 3: $D=90 \%, v=0.5 \mathrm{~m} / \mathrm{min}, T=20 \mathrm{~s}$,

(4) Trial 4: $D=90 \%, v=3 \mathrm{~m} / \mathrm{min}, T=10 \mathrm{~s}$.

Plot water distribution curves along the moving direction (Figure 10),
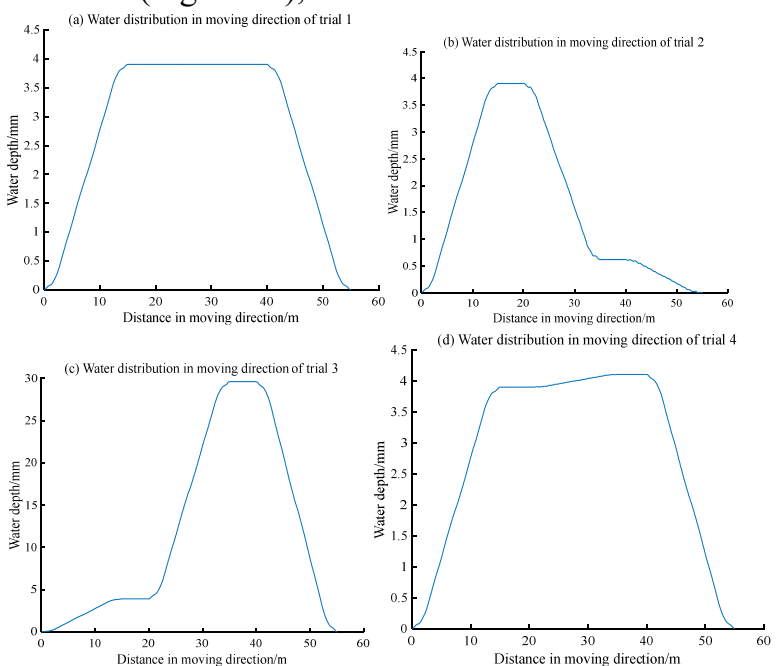

Figure 10. Water distribution in moving direction under different factor combinations.

Compared with Figure 10 (a), Figure 10 (b), (c), (d) show that any variation of duty cycle, speed and PWM period will influence water application. Water application changes significantly with duty cycle and speed but it is not substantially affected by PWM period. Moreover, water application is positively correlated with duty cycle but negatively correlated with speed and PWM period. These conclusions agree with those in section 3.2.1.

Besides water distribution changes linearly in the transition area no matter which factor changes. Therefore the total water application of a management zone should be calculated by integrating the water distribution curve especially when the length of management zone is so short that all the management zone belongs to transition area.

\section{Conclusion}

Theoretical analysis is conducted to find out the relationship between water application and PWM parameters, speed of VRI sprinkler system, spraying feature of single sprinkler for lateral VRI sprinkler system. Based on the analyzing approach, a simulation algorithm is designed and realized with Matlab to simulate the actual spraying process.

Using the water distribution data of single Nelson R3000 sprinkler, a series of experiments are performed which show that water application is proportional to duty 
cycle and inversely proportional to speed of VRI sprinkler system. PWM period only has a little negative relationship with water application. Hence adjusting duty cycle or speed of VRI sprinkler system is still the main approach to change water application.

It is also find that smaller speed or PWM period causes larger error of water depth but better uniformity. Fortunately, compensation method can be adopted to minish the error. Finally experiments also indicate that water distribution pattern in transition area is linear.

\section{Acknowledgment}

This work was supported by National Key R\&D Program of China (No. 2017YFD0700504-2)

\section{References}

1. S.A. O'Shaughnessy, Yenny Fernanda Urrego Pereira, S. R. Evett, et al. Assessing application uniformity of a variable rate irrigation system in a windy location. Applied Engineering in Agriculture, 29(4), 497-510 (2013).

2. W.X. Zhao, J.S. Li, Y.F. Li. Review on variable rate irrigation with continuously moving sprinkler machines. Transactions of the CSAE, 32(13), 1-7 (2016).

3. S. Al-Kufaishi, S. Blackmore, H. Sourell, G. Maletti. Assessment of two variable rate irrigation controllers used on a centre-pivot. Agricultural Engineering International: the CIGR Ejournal, VII, EE 05002 (2005).

4. R.A. Pierce, P.D. Ayers. Evaluation of deposition and application accuracy of a pulse width modulation variable rate field sprayer. Sacramento: 2001 ASAE Annual International Meeting, (2001).

5. W.X. Zhao, J.S. Li, R.M. Yang et al. Field evaluation of water distribution characteristics of variable rate center pivot irrigation system. Transactions of the CSAE, 30(22), 53-62 (2014).

6. Z. Chen, F.Y. Duan, Y.S. Fan, Y.H. Jia, X.Q. Huang. Static simulation on water distribution characteristics of overlap area and whole spraying area for sprinkler. Transactions of the CSAE, 33(16), 104-111 (2017).

7. R.J. Hanks, J. Keller, V.P. Rasmussen et al. Line source sprinkler for continuous variable irrigationcrop production studies. Soil Science Society of America Journal, 40(3), 426-429 (1976).

8. J.E. Christiansen. Irrigation by sprinkling. California Agric. Exp. Station. Bulletin No. 570. Berkeley, Calif.: Univ. of Calif.: (1942)

9. M.D. Dukes, C. Perry. Uniformity testing of a variable-rate center pivot irrigation control systems. Precision Agric, 7(3), 205-218 (2006).

10. Y.S. Zhang, D.L. Zhu, L. Zhang et al. Study on translocating speed and water distribution uniformity of lightweight lateral move irrigation system. Journal of Drainage and Irrigation Machinery Engineering, 32(7), 625-630 (2014).

11. D. K. Giles, D. Downey, T. Kolb, J.G. Grimm. Pulse width modulated sprays for flow rate and droplet size control: spray dynamics and field performance. Pesticide Formulations and Delivery Systems: Meeting the Challenges of the Current Crop Protection Industry, ASTMSTP 1430, (2003).

12. W.P Shi, X. Wang, X.Z. Wang, W.D. Zhuang, C. Wang. Study on variable rate spraying technology based on pulse width modulation and volume control. Journal of Agricultural Mechanization Research, 10, 125-127(2007).

13. X.H. Gong. Study on the water and specific power distribution of the spray-plate sprinkler used in the moving sprinkler machine. Northwest $A$ \& $F$ University, (2015).

14. H.J. Yan. Study on water distribution uniformity of center pivot and lateral move irrigation systems based on variable rate technology. China Agricultural University, (2005).

15. ASABE Standards. 2007. S436.1: Test procedure for determining the uniformity of water distribution of center pivot and lateral move irrigation machines equipped with spray or sprinkler nozzles. St. Joseph, Mich.: ASABE. 